\title{
Introduction to AI Chatbots
}

\author{
Aishwarya Gupta \\ Department of Computer Science \\ Dayananda Sagar College of Engineering \\ Bangalore, India
}

\author{
Divya Hathwar \\ Department of Computer Science \\ Dayananda Sagar College of Engineering \\ Bangalore, India
}

\author{
Anupama Vijayakumar* \\ Assistant Professor \\ Department of Computer Science \\ Dayananda Sagar College of Engineering
}

\begin{abstract}
The modern era of technology has a tremendous impact on the society. With the creation of the ultimate virtual assistants, chatbots have become a popular entity in the conversational services. Chatbots are software programs that use natural language understanding and processing. Chatbots are not just restricted to help the user to complete his tasks such as booking a movie ticket or finding the nearest restaurant, but they also provide a source of entertainment, play a major role in home automation projects, give business strategy tips and help in other ways. In this paper, we will provide an insight into what a chatbot is and the types of chatbots. We also propose a classification based on the current market trends, ease of usability and requirements.
\end{abstract}

Keywords: Chatbot, conversational agent, Artificial Intelligence, Machine Learning, Natural Language Processing, Natural Processing Understanding, ALICE

\section{INTRODUCTION}

Technology plays a massive role in the industry and daily chores. It serves a variety of purposes and is applied in a different way in different parts of the world. Recently, the public has been fantasized by Artificial Intelligence. Artificial Intelligence simulates the cognitive abilities of a human. To be more precise and closely related to humans, the AI Chatbots are now replacing human responses with this software. A Chatbot is a computerized program that acts like a colloquist between the human and the bot, a virtual assistant that has become exceptionally popular in recent years mainly due to dramatic improvements in the areas like artificial intelligence, machine learning and other underlying technologies such as neural networks and natural language processing. These chatbots effectively communicate with any human being using interactive queries. Recently, there's been a massive increase in many cloud-based chatting bot services which have been made available for the development and improvement of the chatbot sector ${ }^{[1]}$ such as IBM Watson, Cleverbot, ELIZA chatbot and many others. These conversational agents have become more responsive and the art of conversation between humans and robots over the past few years have improved drastically. In this paper, we have generalized the AI chatbots and described the general template for the same.

\section{TYPES OF CHATBOTS}

Before the advent of this modern era of technology, manual labor played a major role in all sectors of the industry. With this modern evolution, creation of chatbots has successfully helped in sectors like customer service. However, not all chatbots fall under one category. Chatbots are classified based on the ease of user interface, algorithms and the underlying technologies used. In this paper, (figure 1) it has been proposed that chatbots are mainly categorized into three types.

\section{Menu/Button-Based Chatbots}

The most commonly used and the simplest type of chatbots in the market today are the menu based chatbots, which are in form of buttons and top-down menus. These chatbots follow the principles of decision trees, where you make your decisions to get the ultimate answers. The user is instructed to make these decisions by selecting their options and dig deeper to- wards the appropriate response from the AI. However, these menu-based chatbots are comparatively slower in terms of performance and cannot be completely reliable to get the desired answer.

\section{Keyword Recognition-Based Chatbots}

These chatbots recognizes specific keywords in order to produce a desired result. They listen to what the users enter and respond accordingly. With the help of the AI technology and customized keywords list, the bot determines an appropriate response to the user by using the algorithms. These chatbots will start to fail when there are keyword redundancies between several related questions. For example, if a user asked the question 'How do I set up an auto-login authentication on my phone?', the bot would likely use the keywords like 'auto', 'login', to determine which answer is the best to respond with.

\section{Contextual Chatbots}

Contextual chatbots are one of the most technologically 
advanced bots in the market today. They utilize Machine Learning and Artificial Intelligence technologies like voice recognition, speech-to-text conversion algorithms, etc to interpret the user's sentiments. The underlying ideology of this type of bot is to figure out what the user's intentions are and correspondingly present a thoughtful answer by deciphering the pattern in the database. The bot learns and grows over time by encountering many more experiences. A simple example of such a bot can be seen in a food delivery application. Here the previous order history along with the user's payment options and delivery address are stored on the database. These chatbots analyze the user's perspective and suggest recommendations based on consecutive orders and user's likings.

\section{TEMPLATE OF THE BASIC TAGS}

AIML (Artificial Intelligence Markup Language) is an advanced markup language which is used to create unique chatbots like ALICE, derived from Extensible Markup Language (XML). AIML based chatbot are popular because they are lightweight and easy to configure ${ }^{[2]}$ making it easier to build more artificial intelligent programs. They parse natural language and train the bot using a structured template. There are various different APIs and package with AIML files make this system more flexible and interactive to use in various fields ${ }^{[2]}$.

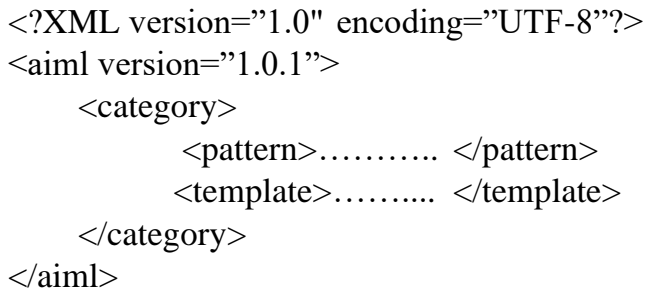

Few other tags that can be used along with the template tags are given below: (Refer Table II)

AIML is a simple pattern language characterized by general inquiries which utilizes AIML tags and formats to interact. The entire process takes place in three parts ${ }^{[3]}$ :

-The user enters his/her query on the chatbot interface

- The user's query is processed to match with the predefined format template

- Pattern matching is done between the knowledge base that stores the predefined template and the user's query in order to arrive at a solution

Finally, this pattern-oriented answer is presented to the user.

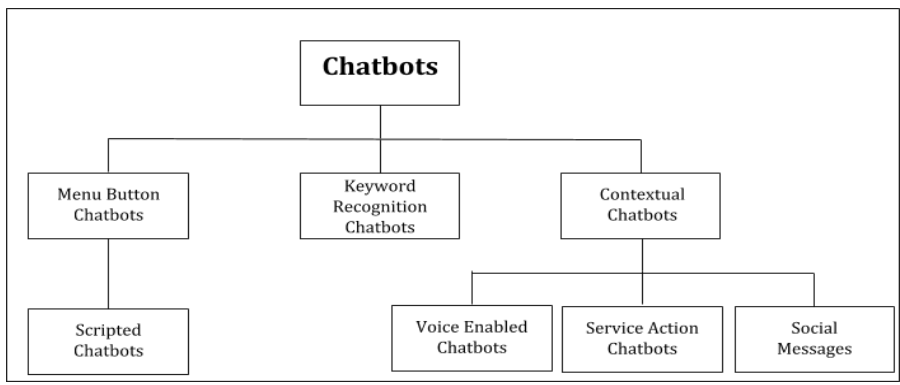

Fig. 1. Proposed Classification of Chatbots

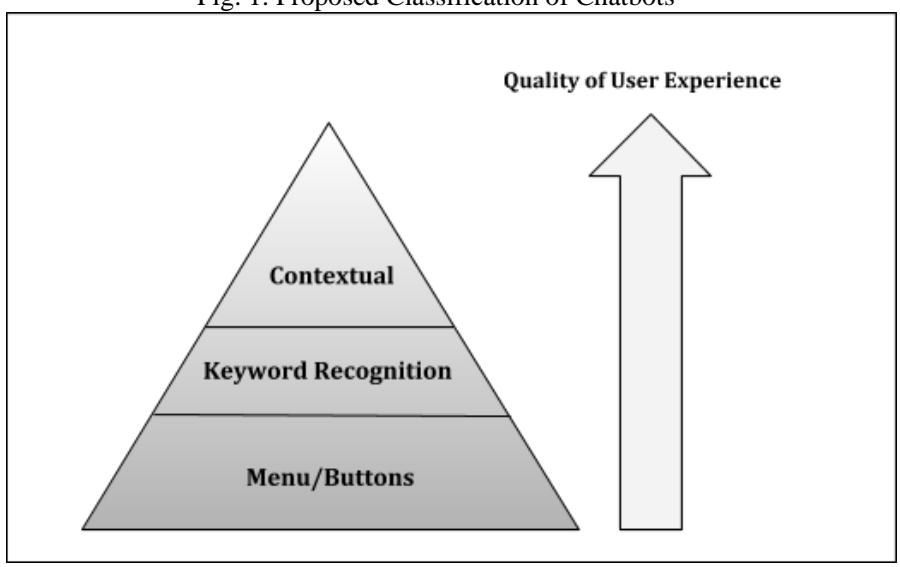

Fig. 2. Preference of Chatbots

\section{APPLICATIONS OF CHATBOTS}

Chatbots are more versatile than given credit for. With the rise of advanced Artificial Intelligence, chatbots have become popular in the past few years as businesses discover innovative ways to put them to use. Being a beneficial agent, chatbots have made life easier for customers as they are available 24/7. They have helped businesses in managing tasks in a customized strategic way. Customer service is one of the major domain where chatbots found a start but their functionality extends much beyond that. Chatbots are communication facilitators which can be integrated into sales \& marketing for lead generation, collecting visitor information \& constantly engaging customers through the lead funnel. Below are some of the different kinds of chatbots that are out in the market currently:

\section{Humorist Chatbot System:}

The main ideology behind the Humorist bot is to make the user laugh. Humorist chatbot is a conver- sational agent that provides a sense of humor by telling humorous anecdotes which are stored in its knowledge base. It is also capable of listening to jokes to interpret the humorous level of a user. It has a set of standard Alice categories that allow holding a general conversation with a user [2]. The chatbot identifies humorous jokes by identifying certain keywords and responds accordingly with funny graphical images and textual responses. 


\section{Dorothy Network Management chatbot:}

Every industry and software application requires network management. Security measures and protocols play an important role in network management to prevent unauthorized access. Dorothy Network Management chatbot is one such bot that takes care of your network via management protocols. Based on ALICE technology, there is

TABLE I

REQUIRED BASIC TAGS

\begin{tabular}{ll}
\hline BASIC TAGS & DESCRIPTION \\
\hline <aiml> & A root tag that indicates the starting and ending of an AIML document. \\
<category> & Forms the basic unit of knowledge and consists of patterns and templates. \\
<pattern> & It is a string intended to correspond to one or more user input and a pattern in matched within this tag. \\
<template> & Specifies the response to an adjusted pattern \\
\hline
\end{tabular}

TABLE II

ADDITIONAL ADD ON TAGS

\begin{tabular}{|c|c|}
\hline <star> & Used when embedded in a <srai > tag, this matches wildcard $*$ character(s) in the <pattern> tag \\
\hline <srai > & Used to match the other categories. When a specific keyword is entered, a simple response is returned. \\
\hline$\langle\mathrm{li}\rangle$ & Used within the <random $>$ tag for random responses from the given list. Specifies a list item. \\
\hline <topic> & $\begin{array}{l}\text { Used as a buffer to store a context so that responses can be based on that context. Usually } \\
\text { used in Binary type conversation and helps AIML to search categories written within the } \\
\text { context of the topic. }\end{array}$ \\
\hline$<$ random> & Used to get random potential set of responses. Enables AIML to respond differently to the \\
\hline <get $>$ & $\begin{array}{l}\text { Sets whatever is within the tags to the variable VALUE. It can be retrieved through the } \\
\text { use of a }<\text { get }>\text { tag. }\end{array}$ \\
\hline <set> & Output whatever is in the variable VALUE. If VALUE has not been <set>, defaults to " ". \\
\hline <think> & Performs whatever is between the tags but not output anything to the user. \\
\hline <condition> & Used to respond to the matching input \\
\hline <that $>$ & Used to respond based on the context. \\
\hline
\end{tabular}

information generated by all modules is processed by the central module and returns to the user through ALICE ${ }^{[4]}$.

\section{Adaptive Modular Architecture based chatbot:}

Another chatbot that is based on ALICE technology is Adaptive Modular Architecture based chatbot. This conversational agent is based on modular knowledge representation and proof-of-concept ${ }^{[2]}$. Knowledge representation plays an integral part of Artificial Intelligence. This modular ontology will help the chatbot response in a more flexible manner. The knowledge representation model is composed of various components: dialogue engine, dialogue analyzer, corpus callosum ${ }^{[2]}$. Corpus callosum plays an important role as it triggers the model and activates only a particular section of the knowledge base at a given period.

\section{Web-based voice chatbot:}

Just like how Alexa is for Amazon, Siri is for apple and cortana is for Microsoft, the web-based voice chatbots have come to light. This Web-based system uses voice recognition technologies to interact with its users. Based on ALICE bot engine, these intelligent digital assistants learn a central module present that collectively receives all the data generated by ALICE which is then processed in order to produce unique results. Another module that holds the entire information is the network history information module ${ }^{[4]}$ which consists of different modules like collect module and history information base which collects the necessary data and store it in the information base respectively ${ }^{[4]}$. All the 
bots aren't "know-it-all" bots instead programmed to act like a real one. Chatbots, unlike other AI tools, will be used to enhance human capabilities and free humans to be more innovative and act upon strategic tactics.

\section{VI.REFERENCES}

[1] A M Rahman, Abdullah Al Mamun, Alma Islam. "Programming challenges of chatbot: Current and future prospective", 2017 IEEE Region 10 Humanitarian Technology Conference (R10-HTC), 2017

[2] Md. Shahriare Satu, Md. Hasnat Parvez, Shamim-Al-Mamun. "Review of integrated applications with AIML based chatbot", 2015 International Conference on Computer and Information Engineering (ICCIE), 2015

[3] Bhavika R. Ranoliya, Nidhi Raghuwanshi, Sanjay Singh. "Chatbot for university related FAQs", 2017 International Conference on Advances in Computing, Communications and Informatics (ICACCI), 2017

[4] Michelle Denise Leonhardt, Liane Tarouco, Rosa Maria Vicari, Elder Rizzon Santos, Michele dos Santos da Silva. "Using Chatbots for Network Management Training through Problem-based Oriented Education", Seventh IEEE International Conference on Advanced Learning Technologies (ICALT 2007), 2007 